\title{
Assimilating Best Track Minimum Sea Level Pressure Data Together with Doppler Radar Data Using an Ensemble Kalman Filter for Hurricane Ike (2008) at a Cloud-Resolving Resolution
}

\author{
XUE Ming ${ }^{1,2,3 *}$ (薛 明) and DONG $\operatorname{Jili}^{1}$ (董继立) \\ 1 Center for Analysis and Prediction of Storms, University of Oklahoma, Norman, OK 73072, USA \\ 2 School of Meteorology, University of Oklahoma, Norman, OK 73072, USA \\ 3 Key Laboratory for Mesoscale Severe Weather/Ministry of Education, and School of Atmospheric Science, \\ Nanjing University, Nanjing 210093, China
}

(Received November 7, 2012; in final form January 18, 2013)

\begin{abstract}
Extending an earlier study, the best track minimum sea level pressure (MSLP) data are assimilated for landfalling Hurricane Ike (2008) using an ensemble Kalman filter (EnKF), in addition to data from two coastal ground-based Doppler radars, at a 4 -km grid spacing. Treated as a sea level pressure observation, the MSLP assimilation by the EnKF enhances the hurricane warm core structure and results in a stronger and deeper analyzed vortex than that in the GFS (Global Forecast System) analysis; it also improves the subsequent 18-h hurricane intensity and track forecasts.

With a 2-h total assimilation window length, the assimilation of MSLP data interpolated to 10-min intervals results in more balanced analyses with smaller subsequent forecast error growth and better intensity and track forecasts than when the data are assimilated every 60 minutes. Radar data are always assimilated at 10 -min intervals.

For both intensity and track forecasts, assimilating MSLP only outperforms assimilating radar reflectivity $(Z)$ only. For intensity forecast, assimilating MSLP at 10-min intervals outperforms radar radial wind $\left(V_{\mathrm{r}}\right)$ data (assimilated at 10-min intervals), but assimilating MSLP at 60-min intervals fails to beat $V_{\mathrm{r}}$ data. For track forecast, MSLP assimilation has a slightly (noticeably) larger positive impact than $V_{\mathrm{r}}(Z)$ data. When $V_{\mathrm{r}}$ or $Z$ is combined with MSLP, both intensity and track forecasts are improved more than the assimilation of individual observation type.

When the total assimilation window length is reduced to $1 \mathrm{~h}$ or less, the assimilation of MSLP alone even at 10-min intervals produces poorer 18-h intensity forecasts than assimilating $V_{\mathrm{r}}$ only, indicating that many assimilation cycles are needed to establish balanced analyses when MSLP data alone are assimilated; this is due to the very limited pieces of information that MSLP data provide.
\end{abstract}

Key words: hurricane, data assimilation, ensemble Kalman filter, best track

Citation: Xue Ming and Dong Jili, 2013: Assimilating best track minimum sea level pressure data together with Doppler radar data using an ensemble Kalman filter for Hurricane Ike (2008) at a cloudresolving resolution. Acta Meteor. Sinica, 27(3), 379-399, doi: 10.1007/s13351-013-0304-7.

\section{Introduction}

Although hurricane track forecasts have improved significantly over the last two decades, hurricane intensity forecasting remains a significant challenge (Cangialosi and Franklin, 2011; Rappaport et al., 2009). The slow improvement in hurricane intensity forecasting is believed to be at least partly due to limited ability to initialize tropical cyclone (TC) vortices accurately in numerical models (Rogers et al., 2006). Convective-scale structures in TCs are believed to have direct or indirect impact on TC intensity and track forecasts (Fovell et al., 2009, 2010; Houze et al., 2007; Wang, 2009).

Techniques for initializing TC vortices include vortex bogusing (e.g., Kurihara et al., 1998; $\mathrm{Pu}$ and

Supported by the United States DOD ONR grants N00014-10-1-0133 and N00014-10-1-0775, NSF grants OCI-0905040 and AGS-0802888, and National Basic Research and Development (973) Program of China (2013CB430103).

${ }^{*}$ Corresponding author: mxue@ou.edu.

(C)The Chinese Meteorological Society and Springer-Verlag Berlin Heidelberg 2013 
Braun, 2001) and direct initialization of TC vortex by assimilating observations on the vortex scale. Recently, a number of studies have demonstrated reasonable success in assimilating airborne or ground-based radar data for initializing TCs, and these studies typically use the three-dimensional variational (3DVAR) (e.g., Du et al., 2012; Pu et al., 2009; Zhao and Xue, 2009; Zhao and Jin, 2008) or ensemble Kalman filter (EnKF) (e.g., Dong and Xue, 2013; Weng and Zhang, 2012; Zhang et al., 2009) methods. While the EnKF method is a theoretically advanced method that makes use of flow-dependent background error covariance derived from a forecast ensemble, Dong and Xue (2013; DX12 hereafter) also found that when starting from the GFS (Global Forecast System) analysis background, it takes 5 to 6 EnKF cycles of 10-min intervals, assimilating full volume radial velocity and reflectivity data from two coastal Doppler radars, to bring the minimum central pressure of a category 3 hurricane to within $5 \mathrm{hPa}$ of the observed best track value. Even with a total of 13 analysis cycles at 10 min apart, the final minimum pressure error remained at nearly $5 \mathrm{hPa}$. Often, the minimum central pressure in TCs is difficult to analyze accurately without direct surface pressure observations.

Best track minimum sea level pressure (MSLP) has been used as observational data and assimilated into numerical models to help improve the TC intensity analysis in recent research studies. Hamill et al. (2011) assimilated the so-called TCVital observations every $6 \mathrm{~h}$ using EnKF in a global forecast model. Here, TCVital is a human-synthesized dataset including the best track estimates of TC minimum central pressure and center location. Despite clearly better track forecasts of TCs in their study compared to operational benchmarks, the resolution of their global model was insufficient to accurately predict the TC intensities; in fact, it was difficult for the global model to maintain the initially intense TCs initialized using TCVital data in their study. Torn and Hakim (2009) and Wu et al. (2010) assimilated hurricane positions in real data studies. Torn (2010) also assimilated best track TC position and MSLP data along with other conventional observations every $6 \mathrm{~h}$ in a mesoscale model at a $36-\mathrm{km}$ horizontal grid spacing using EnKF, although the study did not specifically evaluate the impact of TC position and intensity observations. Earlier, in a proof of concept study, Chen and Snyder (2007) assimilated vortex intensity in terms of maximum vorticity using EnKF in a simple two-dimensional (2-D) barotropic model and found improvement to the vortex intensity and track analysis and forecast. So far, according to our knowledge, the TCVital type of data has not been assimilated into a hurricane prediction model at a cloud-resolving resolution with or without radar data using EnKF. It remains an open question as to the kind of impact one can achieve with TCVital data in such situations. Most recently, Zhao et al. (2012) performed an experiment assimilating MSLP data into a convection-resolving model for a typhoon, in addition to ground-based radar data using a 3DVAR method; the impact of the MSLP assimilation was lost very quickly (in less than $1 \mathrm{~h}$ ) in their study due to the univariate nature of their analysis method - there was no temperature analysis increment to balance the pressure increment in their analyses. Given the multivariate nature of the EnKF method, it is hoped that EnKF can do a much better job in assimilating MSLP data on the convective scale.

In this paper, the impact of assimilating MSLP data into Hurricane Ike (2008) using EnKF is examined. The best track MSLP data are assimilated alone or in addition to the Doppler radar data, with the configurations of the EnKF radar assimilation being the same as in DX12. The rest of this paper is organized as follows. Section 2 introduces the model, observations, and EnKF experiment setup. The analysis increments and the change to the hurricane intensity during the analysis cycles are presented and discussed in Section 3. The impacts of MSLP assimilation on the intensity and track forecasts are discussed in Section 4. Results from several sensitivity experiments are discussed in Section 5, and a summary is provided in Section 6 .

\section{The case, prediction model, observations, and EnKF experiment design}

\subsection{Hurricane Ike and model configurations}

Hurricane Ike (2008) is the third costliest landfalling hurricane in the US history. It made landfall 
near Galveston, Texas at 0700 UTC 13 September 2008. More details of Ike near its landfall can be found in DX12. This study focuses on the analyses and forecasts of Ike shortly before and after its landfall.

The prediction model used in this study is the Advanced Regional Prediction System (ARPS; Xue et al., 2000, 2003). The physical domain is defined by a $515 \times 515 \times 53$ grid with a 4 -km horizontal grid spacing. A vertical grid stretching scheme with a hyperbolic tangent function is used (Xue et al., 1995); the mean vertical grid spacing is $625 \mathrm{~m}$ and the minimum vertical spacing is $50 \mathrm{~m}$ at the surface. The Lin et al. (1983) ice microphysics scheme is used along with the 1.5order TKE-based sub-grid-scale turbulence and PBL parameterizations. Details on these physics options can be found in Xue et al. (2001, 2003). Other details on physics and computational options are the same as those used in DX12.

\subsection{Observations}

The best track MSLP data from the US National Hurricane Center are assimilated between 0400 and 0600 UTC 13 September in the first set of experiments at intervals of either 10 or $60 \mathrm{~min}$ (Table 1). MSLP data, including their values and locations, at such intervals are obtained through linear interpolation between times when best track observations are available. The observed MSLP values changed only slightly within the 2-h data assimilation (DA) window. Radar observations are assimilated alone or together with the MSLP data in the experiments to investigate their relative impacts. As in DX12, radial wind $\left(V_{\mathrm{r}}\right)$ or reflectivity $(Z)$ data from two coastal WSR88D radars at Houston-Gavelston, Texas (KHGX) and Lake Charles, Louisiana $(\mathrm{KLCH})$ are assimilated, always at 10 -min intervals in this study. Details on the radar observations and their assimilation can be found in DX12.

In this study, we treat the best track MSLP data as regular sea level pressure observations located at the best track vortex center location, similar to Hamill et al. (2011). A simple pressure reduction equation (Eq. (1) of Benjamin and Miller (1990)) is applied as the observation operator for the sea level pressure:

$$
P_{\mathrm{SL}}=P_{0}\left(\frac{T_{0}+\gamma z}{T_{0}}\right)^{g / R \gamma}
$$

where $P_{\mathrm{SL}}$ is the sea level pressure, $P_{0}$ and $T_{0}$ are the pressure and temperature at the first model level above the surface, $z$ is the height of the first model level, $\gamma$ is the environmental temperature lapse rate (taken as 9.8 $\mathrm{K} \mathrm{km}^{-1}$ ), $g$ is the gravitational acceleration, and $R$ is the gas constant. A bi-linear horizontal interpolation is used to project the sea level pressure from the model grid onto the best track position. The observational error of MSLP in the human-synthesized TCVital dataset can range from 0.75 to $2 \mathrm{hPa}$ (Tong Mingjing, 2010, personal communication). In this study, the observation error of MSLP is assumed to be $1 \mathrm{hPa}$, smaller than $2 \mathrm{hPa}$ used in Hamill et al. (2011). The

Table 1. List of experiments assimilating MSLP and/or radar observations plus the control experiment

\begin{tabular}{lccc}
\hline Experiment & Assimilated observation type & MSLP DA cycle interval & Assimilation window length \\
\hline CNTL & None & N/A $/ \mathrm{A}$ \\
P60W2h & MSLP & $60 \mathrm{~min}$ & $2 \mathrm{~h}$ \\
P10W2h & MSLP & $10 \mathrm{~min}$ & $2 \mathrm{~h}$ \\
VrW2h & $V_{\mathrm{r}}$ & $\mathrm{N} / \mathrm{A}$ & $2 \mathrm{~h}$ \\
VrP60W2h & $V_{\mathrm{r}} \&$ MSLP & $60 \mathrm{~min}$ & $2 \mathrm{~h}$ \\
VrP10W2h & $V_{\mathrm{r}} \&$ MSLP & $10 \mathrm{~min}$ & $2 \mathrm{~h}$ \\
ZW2h & $Z$ & $\mathrm{~N} / \mathrm{A}$ & $2 \mathrm{~h}$ \\
ZP60W2h & $Z$ \& MSLP & $60 \mathrm{~min}$ & $2 \mathrm{~h}$ \\
ZP10W2h & $Z$ \& MSLP & $10 \mathrm{~min}$ & $2 \mathrm{~h}$ \\
P10W1h & MSLP & $10 \mathrm{~min}$ & $1 \mathrm{~h}$ \\
VrW1h & $V_{\mathrm{r}}$ & N/A & $1 \mathrm{~h}$ \\
VrP10W1h & $V_{\mathrm{r}} \&$ MSLP & $10 \mathrm{~min}$ & $1 \mathrm{~h}$ \\
P10W30m & MSLP & $10 \mathrm{~min}$ & $30 \mathrm{~min}$ \\
VrW30m & $V_{\mathrm{r}}$ & $\mathrm{N} / \mathrm{A}$ & $30 \mathrm{~min}$ \\
VrP10W30m & $V_{\mathrm{r}} \&$ MSLP & $10 \mathrm{~min}$ & $30 \mathrm{~min}$ \\
\hline
\end{tabular}


choice of the relatively small MSLP observation error is partially based on the observation that the analyzed MSLP without MSLP data is always positively biased (i.e., the analyzed hurricane is too weak) in this case, and a smaller MSLP observation error is expected to "push" the minimum pressure closer to the best track observation. Also we note that frequent assimilation of time interpolated best track data has an effect that is somewhat similar to the nudging method, where the model state is "nudged" towards the observations or an analysis persistently over a time period. In our case, the model state is constrained by the best track observations at multiple time levels through EnKF data assimilation. Such frequent assimilation and the associated model adjustments during the assimilation cycles are expected to increase/accelerate the impact of the very limited number of MSLP data within a relatively short period of time. The model grid, the radar locations, and radar data coverage are shown in Fig. 1, along with the best track from 0300 UTC 13 to 0000 UTC 14 September.

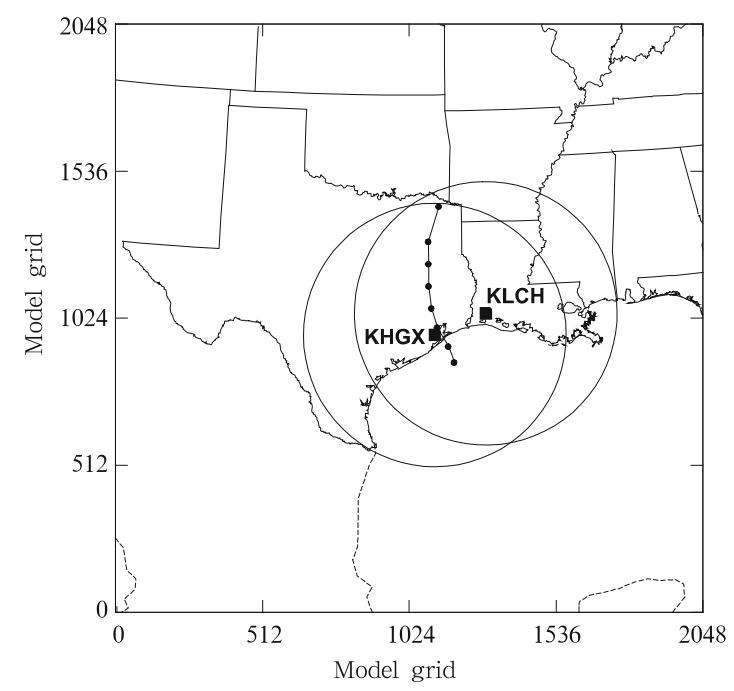

Fig. 1. The model grid, best track (denoted by black dots), and radar coverage for Ike. The positions of two radars are denoted by black squares. The range circles of Houston-Gavelston, Texas (KHGX) and Lake Charles, Louisiana $(\mathrm{KLCH})$ radars are for a maximum range of $460 \mathrm{~km}$. Best track covers 0300 UTC 13 to 0000 UTC 14 September and hurricane locations are plotted every $3 \mathrm{~h}$.

\subsection{EnKF experiment design}

The ensemble square-root filter (EnSRF) of Whitaker and Hamill (2002) forms the basis of our EnKF assimilation system; the initial implementation of the EnSRF for the ARPS system is described in Xue et al. (2006) and the generation of ensemble initial conditions follows DX12.

Briefly, the forecast ensemble is created by adding mesoscale and convective-scale perturbations in two steps. In the first step, a single 4 -h forecast is run from the analysis at 1800 UTC 12 September of GFS of the National Centers for Environmental Prediction (NCEP) interpolated to the $4-\mathrm{km}$ model grid; mesoscale perturbations are added to the 4-h forecast valid at 2200 UTC in the entire model domain to create an ensemble of 32 members. The perturbations are created by smoothing Gaussian random perturbations with zero mean using a $2-\mathrm{D}$ recursive filter (Purser et al., 2003), with a horizontal de-correlation scale of 100 km (e.g., Huang, 2000; Jung et al., 2012). The perturbations are scaled to have standard deviations of 2 $\mathrm{m} \mathrm{s}^{-1}$ for $u$ and $v, 1 \mathrm{~K}$ for $\theta$, and $1 \mathrm{hPa}$ for $p$. For $q_{\mathrm{v}}$, the relative standard derivation is $10 \%$ of the unperturbed value, to avoid excessively large absolute perturbations at the upper levels. Other state variables are not perturbed in this step. Six-hour-long ensemble forecasts are then carried out from these perturbed initial conditions to develop evolved background error covariance structures on the mesoscale.

In the second step, at 0400 UTC 13 September, additional convective-scale perturbations with a smaller horizontal de-correlation scale of $12 \mathrm{~km}$ and a vertical de-correlation scale of $4 \mathrm{~km}$ are added to the ensemble forecast fields, but only in regions where observed $Z$ exceeds $10 \mathrm{dBZ}$. These perturbations are created by applying a fifth-order-correlation smoothing function after Tong and Xue (2008). The standard deviations are $2 \mathrm{~m} \mathrm{~s}^{-1}$ for the wind components, $2 \mathrm{~K}$ for $\theta, 10 \%$ for $q_{\mathrm{v}}$, and $1 \mathrm{~g} \mathrm{~kg}^{-1}$ for all microphysical variables. These perturbations are found to yield best analysis and forecast results for the Ike case through many assimilation experiments in DX12 and are therefore used here too.

The lateral boundary conditions are from the 
6-hourly operational GFS analyses with 3-hourly forecasts interleaved in between, available on the 0.5 degree grid. They are also perturbed by adding mesoscale perturbations created in the same way as in step one. Within ARPS, linear time interpolations are performed between the boundary condition times.

In the first set of experiments (Table 1), MSLP and/or radar observations are assimilated in a 2-h window from 0400 to 0600 UTC 13 September 2008, which is the same as in DX12. After the assimilation, an 18 -h deterministic forecast is carried out from 0600 UTC 13 to 0000 UTC 14 September from the ensemble mean analysis. In a control experiment (CNTL) used for reference, a forecast of the same length is started from GFS 0600 UTC analysis without any additional radar or MSLP data. Schematics for three experiments, corresponding to VrP10W2h/ZP10W2h, VrP60W2h/ZP60W2h, and CNTL in Table 1, are shown in Fig. 2. Table 1 gives a full list of experiments. In the experiment names, letters $\mathrm{Vr}, \mathrm{Z}$, and $\mathrm{P}$ denote the assimilation of $V_{\mathrm{r}}, Z$, and MSLP data, respectively. Numbers " 10 " and " 60 " following letter $\mathrm{P}$ in the names indicate the time interval (in minute) at which MSLP data are assimilated. The number following letter $\mathrm{W}$ denotes the total length of assimilation window in hour or minute. In all cases, radar data assimilation interval is $10 \mathrm{~min}$. For example, in experiment $\mathrm{VrP} 10 \mathrm{~W} 30 \mathrm{~m}, V_{\mathrm{r}}$ and MSLP data are assimilated together within a 30-min assimilation window at 10 -min intervals.

Covariance inflation and localization are applied in the EnKF to alleviate the effects of sampling and other sources of error (e.g., model errors) within the ensemble assimilation system. A horizontal covariance localization radius of $300 \mathrm{~km}$ is used for MSLP assimilation, chosen roughly based on the size of the background vortex. Since there is only one MSLP observation at the analysis time and the MSLP is a vortexscale parameter, a large horizontal localization radius is reasonable to ensure that the impact of the MSLP assimilation extends to the entire vortex. A vertical localization radius of $10 \mathrm{~km}$ for MSLP is determined

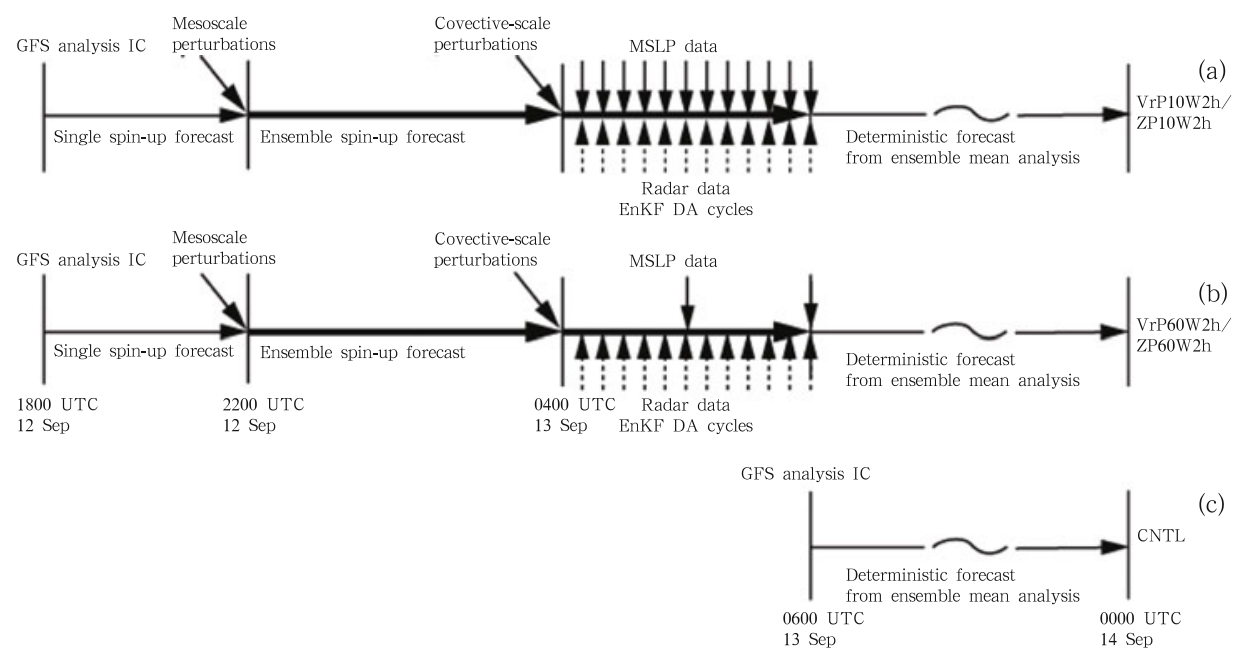

Fig. 2. Schematics showing the flowcharts for experiments that assimilate (a) radar and MSLP data every 10 min for $2 \mathrm{~h}$, (b) radar data every $10 \mathrm{~min}$ but MSLP data every $60 \mathrm{~min}$ for $2 \mathrm{~h}$, and (c) reference forecast NoDA that does not assimilate any radar or MSLP data. The downward arrows denote MSLP DA times and the upward dashed arrows denote radar DA times. Ensemble forecasts are shown as thick horizontal arrows while single deterministic forecasts are shown as thin horizontal arrows. The ensemble DA experiments contain a 4-h single spin-up forecast period starting from GFS analysis at 1800 UTC 12 September 2008, which is followed by a 6-h ensemble spin-up period with mesoscale perturbations added at 2200 UTC 12 September. Convective-scale perturbations are added at 0400 UTC 13 September, the beginning time of EnKF DA cycles. A single deterministic forecast starts from ensemble mean analysis at 0600 UTC 13 September, the ending time of EnKF DA cycles. 
to be close to being optimal through a number of sensitivity experiments; such a radius is reasonable because the surface pressure is directly linked to temperature perturbations in the tropospheric air column. The localization radius for radar observations is $12 \mathrm{~km}$ horizontally and $4 \mathrm{~km}$ vertically, as used in DX12 and is consistent with earlier studies of stormscale EnKF radar data assimilation (Jung et al., 2008; Tong and Xue, 2008). Prior multiplicative covariance inflation of $5 \%$ is applied to the state variables within the regions influenced (through EnKF updating) by the radar and/or MSLP data. Posterior additive covariance inflation is applied to the state variables in regions covered by assimilated radar observations; the magnitudes of the additive perturbations are the same as those used in DX12. In experiments assimilating both radar and MSLP, radar observations are assimilated first, and MSLP data second. Those state variables having the strongest dynamic link to the MSLP observation, including wind components, potential temperature, and pressure, are updated by MSLP. The updating of state variables by radar observations ( $V_{\mathrm{r}}$ and/or $Z$ ) follows DX12. The reflectivity data are used to update only pressure and the mixing ratios of cloud water $\left(q_{\mathrm{c}}\right)$, ice $\left(q_{\mathrm{i}}\right)$, rain water $\left(q_{\mathrm{r}}\right)$, snow $\left(q_{\mathrm{s}}\right)$, and hail $\left(q_{\mathrm{h}}\right)$ while the radial velocity data update all of the eleven state variables, i.e., $u, v$, and $w$, potential temperature $\theta, p$, mixing ratio of water vapor $q_{\mathrm{v}}$, and all microphysical variables. DX12 found that such settings yielded the best hurricane analysis and forecast.

As found in DX12, whenever $V_{\mathrm{r}}$ is assimilated together with $Z$, the analyses and forecasts are very similar to corresponding experiments assimilating $V_{\mathrm{r}}$ only. Thus, for brevity, the experiments assimilating both $V_{\mathrm{r}}$ and $Z$ are not shown in this paper.

\section{Impact of MSLP observations on hurricane analysis}

\subsection{Analysis increments}

The wind and potential temperature analysis increments are plotted in Fig. 3 for P60W2h at 0500 UTC 13 September, the first time that the MSLP data are analyzed in this experiment; this is to illustrate the impact of assimilating MSLP observation. The increment is defined as the difference in the state variables before and after the assimilation of MSLP observation. Figure 3 shows that after analyzing MSLP observation, a strong cyclonic circulation increment around the MSLP data location ( $\mathrm{B}$ in Fig. 3a) is evident at $1 \mathrm{~km}$ above the mean sea level (MSL) (Fig. 3a), indicating an enhancement to the background vortex that is too weak. The center of the increment circulation is not co-located with the background vortex center, determined by the background MSLP (A in Fig. 3a), suggesting that the assimilation of MSLP observation is trying to change the vortex center location as well. The results suggest that the covariance between the pressure and wind fields derived from the ensemble is providing important information to enable the MSLP data to properly influence the wind fields in the EnKF, resulting in dynamically consistent multivariate analyses. A reduction in pressure is also noted at $1 \mathrm{~km}$ above MSL in the vortex region, as shown by the pressure increment in Fig. 3a. The reduction is greater than $10 \mathrm{hPa}$ above the MSLP location, and decreases outward.

The analysis increments of potential temperature at $1 \mathrm{~km}$ above MSL are plotted in Fig. 3b. While the potential temperature increment pattern is more complex than the pressure increment pattern, the increments are all positive in the vortex region, with a maximum value of about $5 \mathrm{~K}$ at this level, indicating that MSLP data assimilation has strengthened the warm core of the cyclone. Positive increments of potential temperature extend upward to the mid troposphere, with an $1-\mathrm{K}$ increment at the 5 -km level above the MSLP data location (Fig. 3d). The 10-km vertical localization radius used allows for the enhancement to the warm core in a deep layer. Increments of wind, pressure, and potential temperature generally decrease with height (figure omitted) as expected, but they do reach the mid troposphere, indicating rather deep vertical correlation between surface pressure and these variables.

Vertical velocity $(w)$ analysis increments, representing changes in regions of updrafts and subsidence 
are plotted along with the background wind vectors in Fig. 3c, for a vertical cross-section through the forecast background vortex center ( $\mathrm{A}$ in Fig. 3a) and the MSLP observation location (B in Fig. 3a). The positive increments of $w$ reflect the enhancement of updraft surrounding the MSLP location. Since the northwest quadrant of the vortex is already over the land at this time and the vortex in that region has started to weaken, the positive increments of $w$ are broader and stronger on the southeast side (right of B in Fig. $3 c)$. There is a region of negative increments inside the eye (almost directly over B, the location of MSLP observation), suppressing the weak updraft found in the background vortex (as indicated by the wind vectors) and correctly establishing descending motion in the eye region. The increments in temperature and wind fields are physically reasonable; they are consistent with the fact that the background vortex is too weak, and the decreased central pressure at 1-km altitude by the MSLP data is accompanied by enhanced warm core and eyewall updrafts as well as downward motion in the eye region.
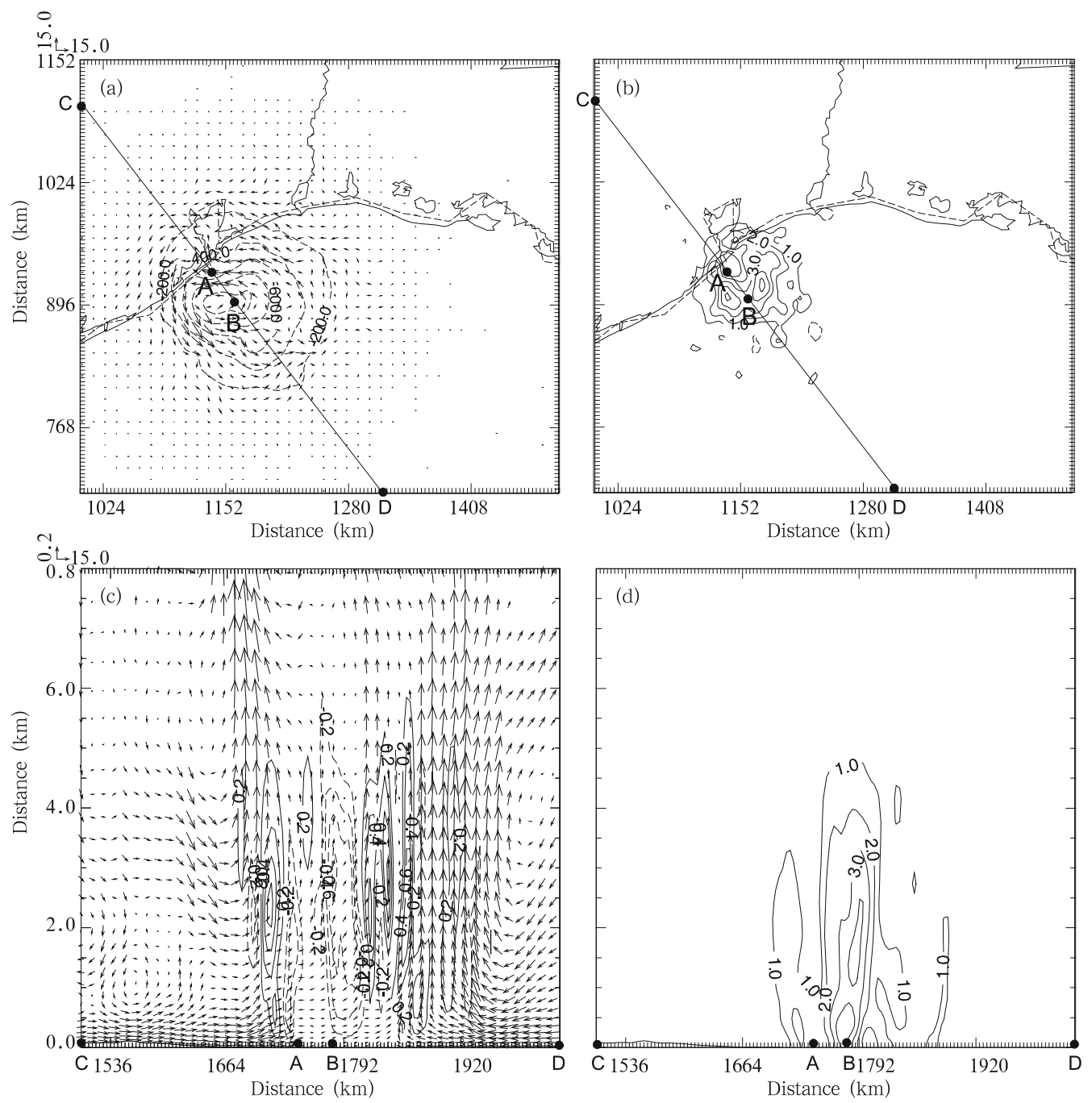

Fig. 3. Increments of (a) horizontal wind component and pressure (every $200 \mathrm{~Pa}$ ) at $1 \mathrm{~km}$, (b) potential temperature (every $1 \mathrm{~K}$ ) at $1 \mathrm{~km},(\mathrm{c})$ vertical velocity $w$ (every $0.2 \mathrm{~m} \mathrm{~s}^{-1}$ ) in the east-west cross-section along line CD in (a), and (d) potential temperature in the same vertical cross-section as (c), at 0500 UTC 13 September of experiment P60W2h. A and $\mathrm{B}$ denote the position of the background vortex center and the position of the MSLP observation, respectively. The background wind vectors are also plotted in (c). 


\subsection{Impact of MSLP data on hurricane inten- sity during the analysis cycles}

To investigate the impact of MSLP data on the analyzed hurricane intensity during the assimilation process, the MSLP values of the analyzed hurricane before and after each analysis are plotted in Fig. 4. The best track MSLP data are linearly interpolated to 10-min interval analysis times for comparison. In our study, the MSLP value at each time is assimilated as a regular surface pressure observation located at the best track vortex center, while the MSLP in the analysis is determined from the analyzed mean sea level pressure field and this analyzed MSLP is not necessarily at the best track vortex center.
We first examine the forecast and analysis MSLPs when only MSLP data are assimilated. When MSLP observations are assimilated at 60-min intervals in P60W2h over a 2-h window (at hours 1 and 2 into the window), the analyzed MSLP decreases by about $14 \mathrm{hPa}$, to $957 \mathrm{hPa}$, during the first analysis at 0500 13 UTC September (Fig. 4a). In the forecast over the next hour without additional data assimilation, the model MSLP increases to $964 \mathrm{hPa}$ by 0600 UTC. The second analysis of MSLP data at 0600 UTC reduces the model MSLP to $954 \mathrm{hPa}, 3 \mathrm{hPa}$ higher than the best track observation.

When MSLP is assimilated at 10-min intervals in P10W2h, the analyzed MSLP is decreased by more
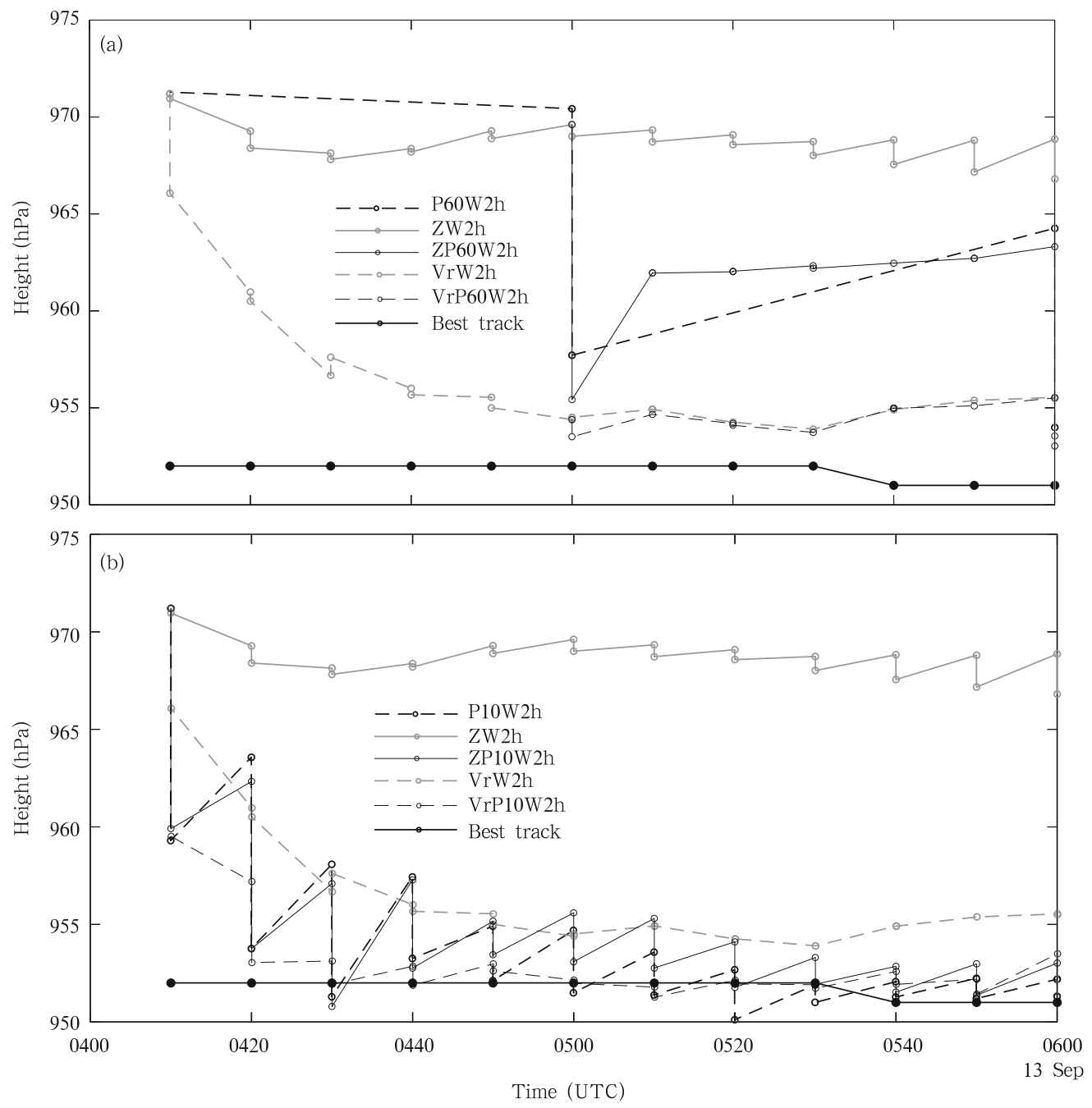

Fig. 4. The analyzed and forecast MSLP during the assimilation cycles of different experiments with (a) 60-min and (b) 10-min assimilation intervals for MSLP, compared to the best track. 
than $10 \mathrm{hPa}$ during each of the first two analysis cycles (Fig. 4b). Over the 2-h assimilation window, the assimilation of MSLP always reduces the analyzed MSLP and the magnitude of reduction decreases gradually with cycle as the model MSLP becomes closer to the observed values. There is always an increase in MSLP in the ensuing forecast, but the amount of increase decreases with the number of cycles, suggesting an increasing level of consistency among model state variables as the model state is continually adjusted through the cycles. At 0520 UTC, the analyzed MSLP actually becomes about $2 \mathrm{hPa}$ lower than the observed value interpolated to this time. Since the analyzed MSLP is not necessarily at the same position as the MSLP observation, this "over-correcting" behavior in $\mathrm{P} 10 \mathrm{~W} 2 \mathrm{~h}$ at $0520 \mathrm{UTC}$ is possible because of unreliable spatial covariance causing under-shooting of analyzed pressure away from the MSLP data location. At the end of the assimilation window, the analyzed MSLP in P10W2h is almost exactly the same as the best track value of roughly $951 \mathrm{hPa}$. Therefore, frequent assimilation of MSLP observations at 10-min intervals is able to improve the intensity of the model TC in terms of MSLP, making it approach the best track intensity after 6 assimilation cycles.

Next, we examine the cases when $V_{\mathrm{r}}$ data are assimilated alone, or together with MSLP data. In experiment VrW2h that assimilates $V_{\mathrm{r}}$ data only at 10 min intervals, the MSLP is reduced by about $5 \mathrm{hPa}$ in the first analysis at 0410 UTC, while in later cycles the decrease by analysis is small and occasionally negative (e.g., at 0430 UTC). Much of the MSLP reduction is achieved during the forecast step in the earlier cycles, indicating that the model pressure field is adjusting to the improved wind field, not surprisingly because of the assimilation of a large number of $V_{\mathrm{r}}$ data (Fig. 4a). In VrP60W2h, the addition of MSLP observation at 0500 UTC further decreases the MSLP by about 1 $\mathrm{hPa}$ compared to VrW2h (Fig. 4a), but the impact is small in the ensuing cycles. At 0600 UTC, the second assimilation of MSLP data results in a final analyzed MSLP in VrP60W2h that is about $2 \mathrm{hPa}$ lower than in VrW2h. Therefore, the impact of assimilating MSLP data at hourly intervals when Doppler radial velocity data from two radars are assimilated at 10-min intervals, in terms of the analyzed MSLP, is minimal in this case.

In VrP10W2h, the MSLP data are also assimilated at 10 -min intervals together with $V_{\mathrm{r}}$ data, the analyzed MSLPs are always lower than those of VrW2h, and MSLP errors generally grow slower during the forecast step than in P10W2h (Fig. 4b). In VrW2h, the surface pressure reduction is achieved almost entirely through model adjustment while in $\mathrm{VrP} 10 \mathrm{~W} 2 \mathrm{~h}$, the assimilation of MSLP provides direct help, resulting in much faster pressure reduction and more accurate final analysis of MSLP (Fig. 4b). In P10W2h, while the MSLP is reduced similarly as in VrP10W2h, the error growth is much larger in the forecast steps, especially during the earlier cycles; this is because of the larger mutual adjustments among the pressure, wind, and other state variables when no other direct observations are assimilated.

We now look at the cases when $Z$ (instead of $V_{\mathrm{r}}$ ) data are assimilated alone or together with MSLP data. In experiment ZW2h, the model MSLP is changed little by the $Z$ assimilation before 0540 UTC (Fig. 4a). Reductions of 2 to $3 \mathrm{hPa}$ in MSLP are achieved in the last three analysis cycles, likely a result of improved cross-covariance between the microphysical and pressure fields in the ensemble (as discussed in DX12). In ZP60W2h, MSLP is assimilated at 0500 and $0600 \mathrm{UTC}$; at 0500 UTC, an additional MSLP reduction of $14 \mathrm{hPa}$ is achieved by assimilating MSLP data (Fig. 4a). While the storm weakens quickly to approximately $963 \mathrm{hPa}$ in MSLP during the subsequent 10-min forecast, the MSLP in the forecast is much lower than that of ZW2h, and remains so until the end of the assimilation window. Between 0510 and 0600 UTC, the assimilation of $Z$ data every 10 min causes very little change to MSLP, and it remains more or less constant; at 0600 UTC, the second analysis of MSLP data further reduces the minimum pressure by $10 \mathrm{hPa}$ to reach $953 \mathrm{hPa}$, only $3 \mathrm{hPa}$ higher than the observed. Still, relatively rapid error growth in the subsequent forecast is expected with the very limited number of MSLP analysis cycles in this case; this fact will be discussed further later. 
The MSLP values in ZP10W2h are very similar to those of P10W2h before 0450 UTC (Fig. 4b). In the last few cycles, the analyzed MSLP in ZP10W2h is closer to that of best track, and slightly higher than that of P10W2h. Comparing ZP10W2h and P10W2h indicates that the assimilation of $Z$ in addition to MSLP does not seem to further improve the intensity analysis. During some of the cycles (e.g., 0450-0530 UTC), the analysis and forecast MSLP in ZP10W2h are slightly worse than $\mathrm{P} 10 \mathrm{~W} 2 \mathrm{~h}$ while in some other cycles (e.g., 0520 UTC), P10W2h over-analyzes the intensity somewhat. But in general, the assimilation of MSLP data every $10 \mathrm{~min}$ with and without $Z$ data results in analyzed MSLP values that are close to the best track data after several analysis cycles (Fig. 4b). Given the apparent effectiveness of MSLP data in analyzing the minimum surface pressure of a hurricane, does it mean that we no longer need radar or other types of high-resolution observations? We will examine the analyzed hurricane structures next, which will help answer this question.

\subsection{Analyzed hurricane structures}

Figure 5 shows the wind, sea level pressure, and temperature fields of GFS analysis, and the final EnKF analyses of VrW2h, P10W2h, and VrP10W2h at the surface and in the west-east vertical crosssections through the individual vortex center of each experiment. For brevity, only experiments with MSLP assimilated every $10 \mathrm{~min}$ are shown here. With $V_{\mathrm{r}}$ data from two coastal radars assimilated, the hurricane in VrW2h (Figs. 5c and 5d) is much stronger than that in the GFS analysis (Figs. 5a and 5b), with both lower center pressure and larger radial pressure gradient. In all three DA experiments, and in the GFS analysis, the maximum wind is always on the east side of the vortex. The vortex of VrW2h is much deeper than that of the GFS analysis, as shown by the strong wind exceeding $48 \mathrm{~m} \mathrm{~s}^{-1}$ extending to $6.4 \mathrm{~km}$ vertically (Fig. $5 \mathrm{~d})$. The maximum wind in VrW2h is approximately $59.5 \mathrm{~m} \mathrm{~s}^{-1}$, which is much larger than the $44.5 \mathrm{~m} \mathrm{~s}^{-1}$ in GFS analysis. The warm core is also much stronger and deeper in VrW2h than in the GFS analysis. When $Z$ is assimilated in $Z W 2 \mathrm{~h}$, the final analyzed storm is also stronger than in the GFS analysis, but weaker than in P10W2h (figure omitted here; cf. DX12 Figs. $6 \mathrm{~b}, 6 \mathrm{f}$, and $6 \mathrm{j})$.

In $\mathrm{P} 10 \mathrm{~W} 2 \mathrm{~h}$, the minimum central pressure is even lower than in VrW2h (Fig. 5e). The wind field of $\mathrm{P} 10 \mathrm{~W} 2 \mathrm{~h}$, having a maximum of $55.7 \mathrm{~m} \mathrm{~s}^{-1}$, is stronger and deeper than that of GFS analysis, but weaker than that of VrW2h (Fig. 5f). Strong winds of greater than $48 \mathrm{~m} \mathrm{~s}^{-1}$ extend to around $3.6 \mathrm{~km}$ above the surface in P10W2h (Fig. 5f), shallower than that in VrW2h (Fig. 5d). The warm core in $\mathrm{P} 10 \mathrm{~W} 2 \mathrm{~h}$ is stronger than in the GFS analysis, but not as deep as in VrW2h. It is clear that the assimilation of MSLP data is more effective in reducing the surface pressure while the assimilation of $V_{\mathrm{r}}$ data is more effective in establishing the strong vortex circulation.

The analyzed minimum central pressure of VrP10W2h is close to that of P10W2h and lower than that of VrW2h (Fig. 5g), and its vortex is stronger and deeper than in VrW2h with a maximum speed of $61.7 \mathrm{~m} \mathrm{~s}^{-1}$ and the region with wind speed exceeding $48 \mathrm{~m} \mathrm{~s}^{-1}$ extends to $7 \mathrm{~km}$ above the surface (Fig. 5h). The warm core in VrP10W2h is as deep as in VrW2h. In ZP10W2h, the final analysis is similar to P10W2h (figure omitted).

The right column of Fig. 5 also shows the temperature field in the vertical cross-sections. It is clear that VrW2h (Fig. 5d) and VrP10W2h (Fig. 5h) produce more realistic warm-core temperature structures than in P10W2h (Fig. 5f); this is perhaps not too surprising because MSLP data do not directly provide information on the vertical structures of the hurricane.

In Fig. 5, the vortex structure in $\mathrm{P} 10 \mathrm{~W} 2 \mathrm{~h}$ appears relatively smooth; by comparison, more convective-scale structures are analyzed when $V_{\mathrm{r}}$ observations are assimilated and such convective-scale structures can be important for hurricane prediction. The assimilation of MSLP does help strengthen the vortex although it tends not to introduce convectivescale structures. When both types of observations are assimilated together, the hurricane acquires a lower central pressure and stronger and deeper vortex circulations than when $V_{\mathrm{r}}$ or MSLP is assimilated individually. 

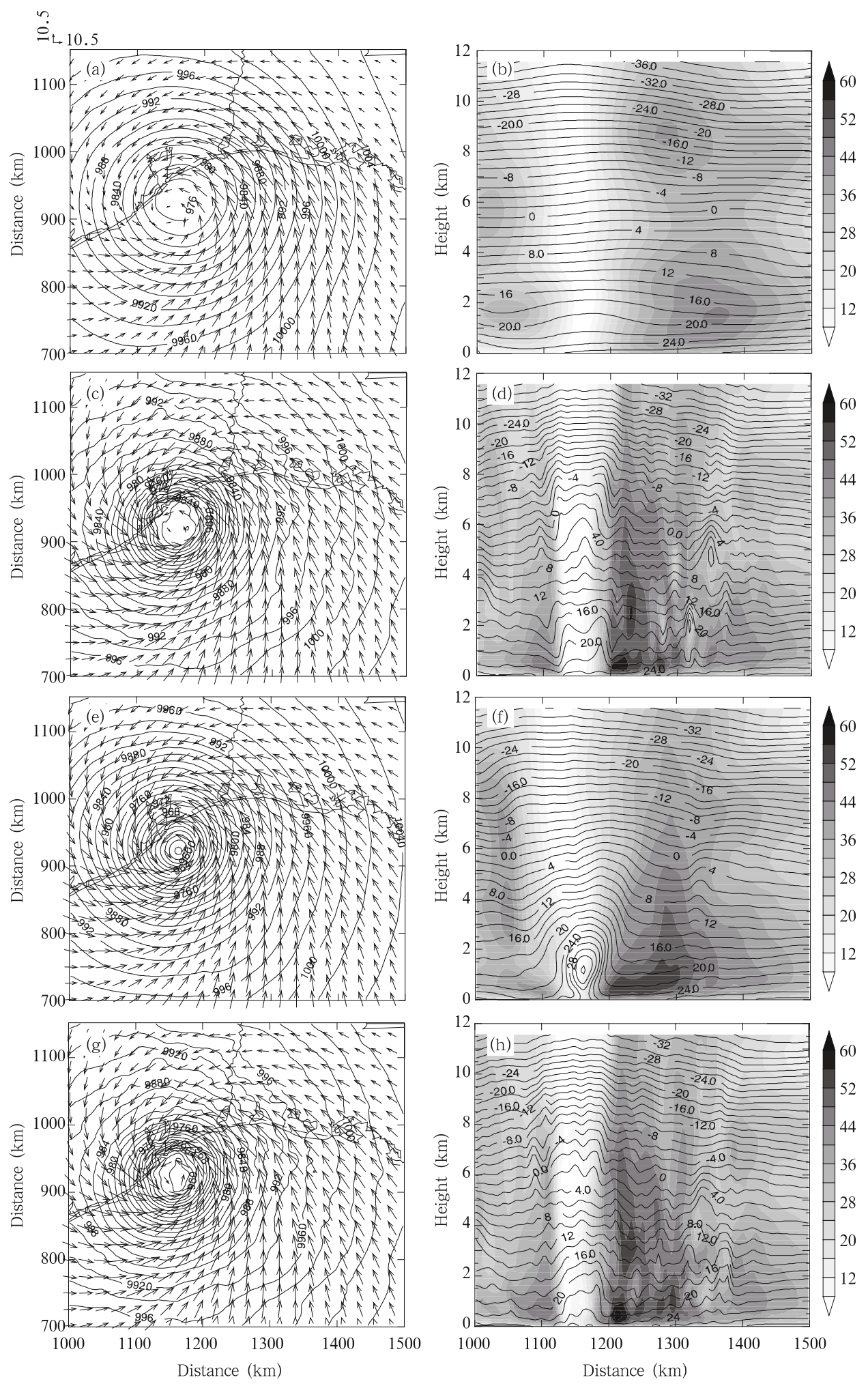

Fig. 5. The analyzed surface horizontal wind speed (shaded) and sea level pressure at 0600 UTC 13 September from (a) GFS analysis, (c) VrW2h, (e) P10W2h, and (g) VrP10W2h. (b), (d), (f), and (h) show the east-west cross-section of horizontal wind and temperature through the individual vortex center of each experiment. 
Individual cross-section shown earlier may not be representative of the vortex structure. Azimuthally averaged radius-height wind fields and temperature anomalies of the final analysis at 0600 UTC are plotted in Fig. 6, together with those of the GFS analysis. The azimuthal-mean horizontal winds in all three DA experiments are substantially stronger than those in the GFS analysis. In P10W2h, the maximum azimuthalmean wind exceeds $42 \mathrm{~m} \mathrm{~s}^{-1}$ while the maximum temperature anomaly is over $12^{\circ} \mathrm{C}$ and is located at $1-2 \mathrm{~km}$ above the surface (Figs. 6e and 6f). The latter very
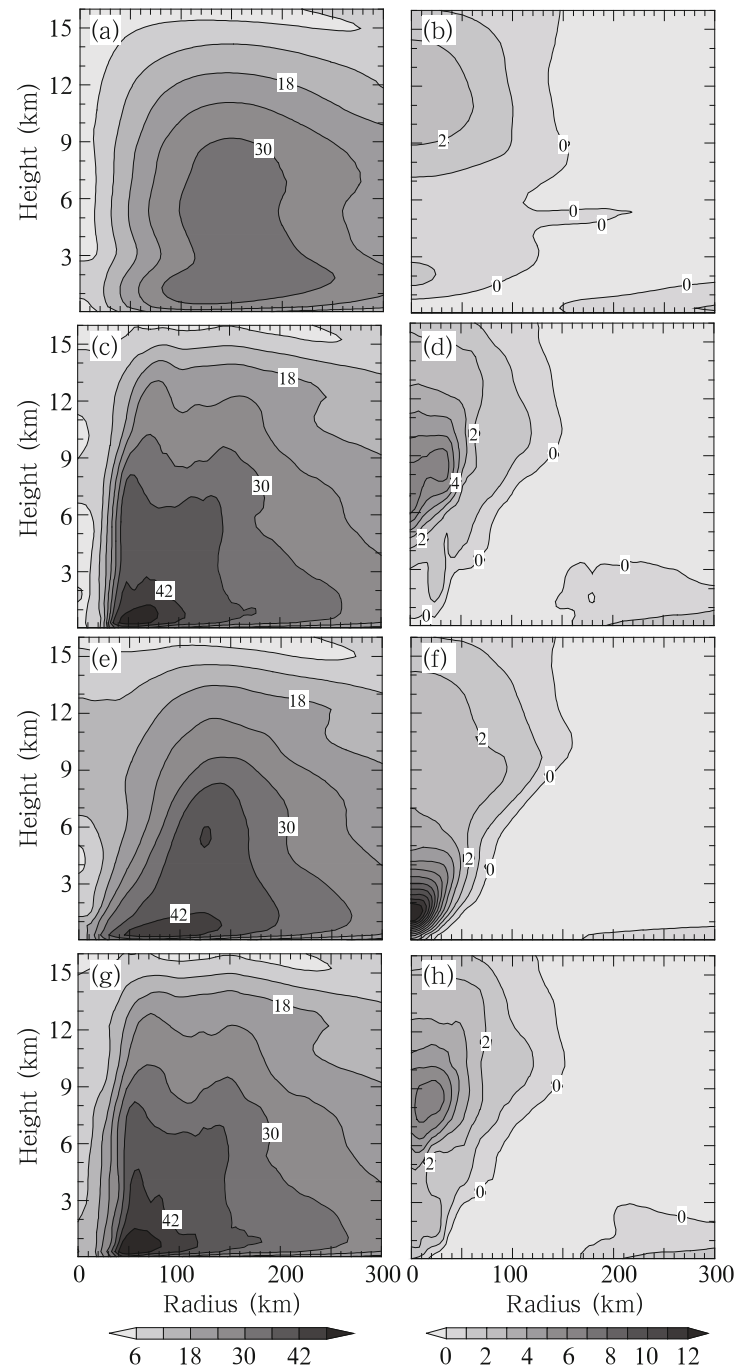

Fig. 6. Azimuthally averaged radius-height plots of horizontal winds from (a) GFS analysis, (c) VrW2h, (e) P10W2h, and (g) VrP10W2h. (b), (d), (f), and (h) show the radius-height plots of temperature anomaly of each experiment. strong temperature anomaly centered in the lower troposphere is not consistent with commonly observed or simulated structures within TCs (Emanuel, 2005; Wang, 2002) where the largest anomaly is usually found in the mid-troposphere. With MSLP data being the only data source in this case, the vertical spreading of observational information depends strongly on the spatial error covariance, which tends to be less reliable than direct observations; this may be the reason for the unrealistically-strong warm core at the low levels found in P10W2h. In VrW2h, the horizontal wind fields have much larger horizontal gradients in the eyewall region than in the GFS analysis, with roughly vertical isotachs in the eyewall and a maximum wind of over $48 \mathrm{~m} \mathrm{~s}^{-1}$ (Figs. 6c and 6d). The maximum temperature anomaly is about $6^{\circ} \mathrm{C}$ and is located at $6-8 \mathrm{~km}$ above the surface, a structure that is more consistent with typical observed TC structures. In VrP10W2h, the structures of horizontal winds and temperature anomaly are similar to those of VrW2h, except that the region with wind speeds exceeding $42 \mathrm{~m} \mathrm{~s}^{-1}$ is about $2.5 \mathrm{~km}$ deeper than in VrW2h (Figs. $6 \mathrm{~g}$ and $6 \mathrm{~h}$ ). In general, the assimilation of $V_{\mathrm{r}}$ and/or MSLP data significantly enhance the axisymmetric vortex circulation and warm core structure. The vortex structure obtained by assimilating $V_{\mathrm{r}}$ data is more realistic than assimilating MSLP only and assimilating both $V_{\mathrm{r}}$ and MSLP data gives the strongest hurricane vortex.

\subsection{Sensitivity to model variable updating in EnKF}

During the assimilation of MSLP, the model state variables such as pressure, wind, and potential temperature are updated. We examine here how the impacts of state variable updating evolve with time, which will help us understand which model variables are most important in maintaining the storm strength during the analysis-forecast cycles and how the model adjusts to the updated fields in the subsequent forecast. Towards this goal, a set of experiments is performed based on experiment P60W2h. In these experiments, MSLP assimilation is performed at 0500 UTC using the ensemble forecast background of P60W2h, but each experiment updates only a single or a subset of state variables. A 20-min forecast is launched 
from the ensemble mean analysis from each of the experiments, which will be compared to the forecast launched from the ensemble mean forecast at 0500 UTC, essentially from the forecast background without further DA. The model variable updated by MSLP is indicated by the experiment name: ExpT only updates potential temperature while ExpWTP updates wind, potential temperature $(\theta)$, and pressure. The minimum value of surface pressure difference between the control forecast and the forecasts with MSLP DA (surface pressure forecast with DA minus

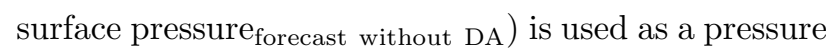
impact index (PI) to measure the impact of DA on the model surface pressure. A more negative PI indicates a greater impact, i.e., the forecast storm is stronger.

The PIs of the experiments are plotted in Fig. 7 at 0,10 , and 20 min after the DA time. At the initial time (0500 UTC), the PIs of the experiments in which pressure is updated by the MSLP data (ExpP, ExpTP, and ExpWTP) are all about $-14.3 \mathrm{hPa}$, but are zero in all other experiments (since the pressure is not updated). After $10 \mathrm{~min}$, the large impact on pressure in $\operatorname{ExpP}$ decreases to $-0.3 \mathrm{hPa}$, and to $-0.15 \mathrm{hPa}$ by 20 min, indicating that the initial impact from MSLP DA is almost completely lost in this case. Clearly, updating pressure only when assimilating MSLP data is ineffective; this is consistent with the results of Zhao et al. (2012) where univariate analysis of the MSLP data using the 3DVAR method was also found ineffective.

The PIs of ExpW and ExpT increase from zero at the initial time to -5.1 and $-2.3 \mathrm{hPa}$, respectively, at $10 \mathrm{~min}$ of forecast, clearly due to the adjustment of model pressure to the wind or potential temperature field updated by the MSLP data. Updating the model wind field appears to have a larger impact than updating $\theta$ on the forecast pressure. By $20 \mathrm{~min}$, the PI of ExpW increases slightly (more negative) while that of ExpT decreases slightly, but the changes are less than $1 \mathrm{hPa}$ in both cases. Updating both wind and $\theta$ in ExpWT leads to a more negative PI during the forecast than updating wind or $\theta$ individually, giving PIs of -6.7 and $-6.9 \mathrm{hPa}$ at 10 - and $20-\mathrm{min}$ forecast times, respectively, and these values are very close to those of ExpWTP. At the same time, the PIs of ExpTP and ExpT are very similar at the corresponding times (Fig. 7) while the PIs of ExpWP (figure omitted) are very similar to those of ExpW. These results indicate that even though MSLP contains pressure information, the updating of wind and temperature fields when using the data is much more important than updating pressure itself; in fact, updating pressure itself has little impact on the subsequent forecasting with or without updating other state variables; the improvement to the analyzed MSLP is in a sense superficial in absence of support of other state variables. In the model, the pressure field adjusts quickly to the temperature and wind fields, apparently through hydrostatic and gradient wind balance adjustments. Such results also point to us the importance of cross-variable covariance derived from the ensemble, which is responsible for the EnKF updating of state variables rather than pressure when assimilating the MSLP data.

\section{Impact on forecast}

\subsection{Intensity forecast}

The 18-h forecast MSLPs for all DA experiments are plotted in Fig. 8, along with those of CNTL forecast, and they are compared to the best track data. The best track MSLP is $951 \mathrm{hPa}$ at 0600 UTC 13 September, one hour before landfall; after landfall, the MSLP gradually increases, and it is $980 \mathrm{hPa}$ by 0000 UTC 14 September (Fig. 8). Overall, all of the DA experiments predict lower MSLPs than CNTL before

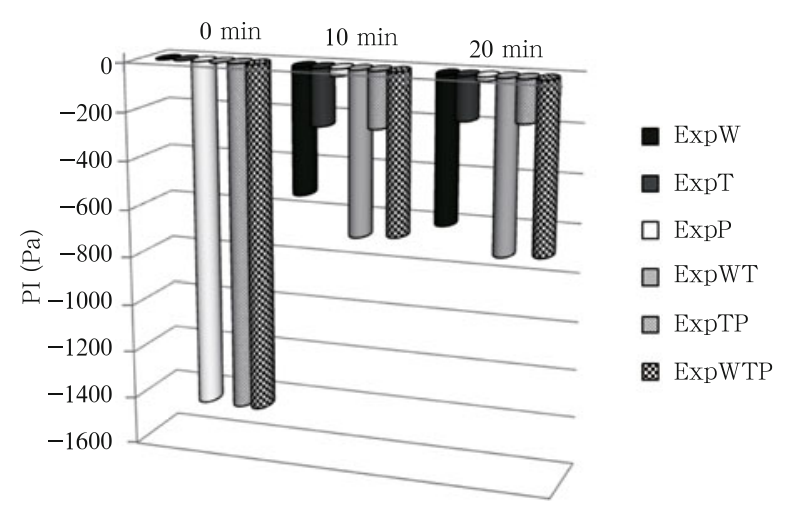

Fig. 7. Pressure impact index (PI; Pa) with time for experiments updating various model variables. 

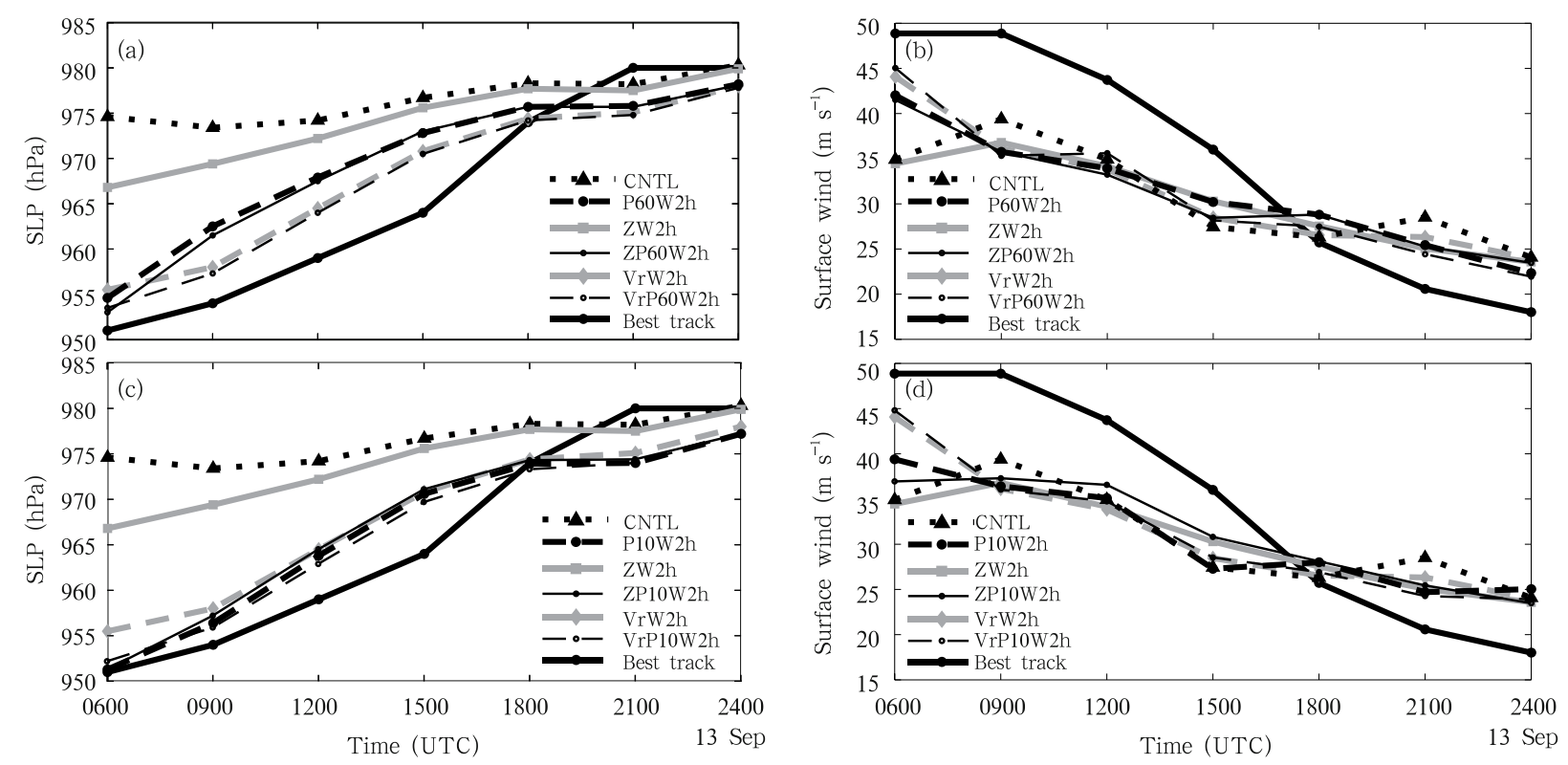

Fig. 8. Forecast $(\mathrm{a}, \mathrm{c})$ minimum sea level pressures and (b, d) surface maximum wind with time, compared to the observed best track and CNTL.

1800 UTC. The slower weakening between 1500 and 2100 UTC compared to the best track might be a result of model errors (DX12), leading to somewhat too strong storms after 1800 UTC.

The MSLP of P60W2h at 0600 UTC is around $955 \mathrm{hPa}$, slightly lower than that of VrW2h (Fig. 8a). However, the MSLP of P60W2h increases much faster than the best track and VrW2h during the first 3-h forecast, and is $5 \mathrm{hPa}$ higher than VrW2h at 0900 UTC. During the following forecast, the MSLPs of $\mathrm{P} 60 \mathrm{~W} 2 \mathrm{~h}$ are always higher than those of VrW2h. The MSLP of $\mathrm{VrP} 60 \mathrm{~W} 2 \mathrm{~h}$ is $2 \mathrm{hPa}$ lower than that of VrW2h at 0600 UTC, but becomes similar afterwards. When $Z$ is assimilated in addition in $\mathrm{ZP} 60 \mathrm{~W} 2 \mathrm{~h}$, the final analyzed MSLP is slightly lower than in P60W2h but the predicted MSLPs become similar after 0900 UTC.

With MSLP data assimilated every $10 \mathrm{~min}$ in P10W2h (Fig. 8c), the final analyzed MSLP is quite close to the best track. The predicted MSLP is similar to that of VrW2h after 0600 UTC, and is higher than the best track before 1800 UTC. All three experiments assimilating MSLP at 10-min intervals are quite similar to one another, with differences in MSLP being always smaller than $2 \mathrm{hPa}$. Starting with a slightly higher MSLP than the other two at 0600 UTC, the intensity forecast of $\mathrm{VrP} 10 \mathrm{~W} 2 \mathrm{~h}$ during the 18-h forecast is generally the best among all the DA experiments conducted in this study in terms of MSLP.

It appears that the assimilation of MSLP at 60min intervals is insufficient to establish a strong and well-balanced hurricane. MSLP error in P60W2h increases quickly during the first several forecast hours. More frequent MSLP assimilation at 10-min intervals leads to a more balanced vortex and slower error growth during subsequent forecast.

The assimilation of MSLP at 10- and 60-min intervals in addition to $Z$ data significantly improves the intensity forecast of ZW2h. Since the MSLP forecast of $\mathrm{VrW} 2 \mathrm{~h}$ is already close to the best track, frequent MSLP assimilation at 10-min intervals is necessary to achieve further noticeable improvement.

The maximum surface winds of forecasts in the experiments are plotted in Figs. 8b and 8d, and are compared to those of best track. At 0600 UTC, the analyzed maximum winds of the experiments assimilating radar and/or MSLP data are all stronger than those of CNTL, except that of ZW2h, which is similar. Experiments assimilating both $V_{\mathrm{r}}$ and MSLP (i.e., VrP60W2h in Fig. 8b and VrP10W2h in Fig. 8d) 
always have the strongest analyzed maximum wind, indicating the benefit of assimilating both $V_{\mathrm{r}}$ and MSLP observations. Although the analyzed maximum winds in $\mathrm{P} 10 \mathrm{~W} 2 \mathrm{~h}$ and $\mathrm{ZP} 10 \mathrm{~W} 2 \mathrm{~h}$ are weaker than those in $\mathrm{P} 60 \mathrm{~W} 2 \mathrm{~h}$ and $\mathrm{ZP} 60 \mathrm{~W} 2 \mathrm{~h}$, respectively, the forecasts of P10W2h and ZP10W2h at 0900 UTC show stronger winds than their counterparts assimilating MSLP at 60-min intervals. This is more or less consistent with the MSLP forecasts, where the assimilation of MSLP at 10-min intervals helps to build up a more dynamically-balanced vortex, and the impact from DA lasts longer than when MSLP is assimilated at 60-min intervals. Generally, the maximum surface wind forecasts are close to one another in all experiments after 0900 UTC. Note that the maximum wind can be affected by localized convective activities while the MSLP tends to be a system-integrated more reliable measure of the vortex intensity (Zhu and Zhang, 2006).

In conclusion, with frequent assimilation of MSLP data in a cloud-resolving model, hurricane intensity forecast can be improved; such improvement was not clearly achieved in Hamill et al. (2011) when assimilating the TCVital data into a coarser resolution global model at a much lower frequency. The high assimilation frequency is necessary to achieve sustained impacts.

MSLP assimilation at 10-min intervals outperforms $V_{\mathrm{r}}$ assimilation in terms of the MSLP forecast. The MSLP parameter measures mainly the overall vortex intensity, but does not necessarily represent well sub-vortex convective-scale structures in a hurricane. For this reason, we further verify the wind forecasts of CNTL, VrW2h, P10W2h, and VrP10W2h against the $V_{\mathrm{r}}$ observations from the two coastal radars from 0600 through 0900 UTC when Ike was near the coast. The root-mean-square differences (RMSD) between model predicted and observed $V_{\mathrm{r}}$ are plotted in Fig. 9. Such calculations are limited to regions where observed reflectivity exceeds $10 \mathrm{dBZ}$.

All DA experiments show clear improvement over CNTL when verified against $V_{\mathrm{r}}$ (Fig. 9). The RMSD against KHGX radar in CNTL grows rapidly partly because the vortex of CNTL moves slower than the best track and other DA experiments from 0600 to 0900 UTC (Fig. 10a). The RMSD values for VrW2h are $34 \%$ and $60 \%$ of those for P10W2h at 0600 UTC when verified against KHGX and $\mathrm{KLCH}$ radars, respectively (Fig. 9). The VrW2h analyses fit the observed $V_{\mathrm{r}}$ data reasonably well, whereas the analyzed winds assimilating MSLP data only match the observed $V_{\mathrm{r}}$ data much worse. After $2 \mathrm{~h}$ of forecast, the RMSD values of VrW2h and P10W2h become closer as the RMSD of P10W2h decreases with time while that of VrW2h increases with time; their differences are less than $1 \mathrm{~m} \mathrm{~s}^{-1}$ by $3 \mathrm{~h}$ for both radars. These indicate that wind errors are reduced during the forecast as the wind field adjusts to the improved vortex due to MSLP DA, while wind errors that are reduced by the assimilation of $V_{\mathrm{r}}$ data increase as the forecast error grows in general.

In a short range forecast ( $\sim 3 \mathrm{~h}$ in our study), the assimilation of radar observations appears to have an advantage over the assimilation of MSLP observations on the convective scale (as observed by radar data). Combining $V_{\mathrm{r}}$ and MSLP data clearly gives the best
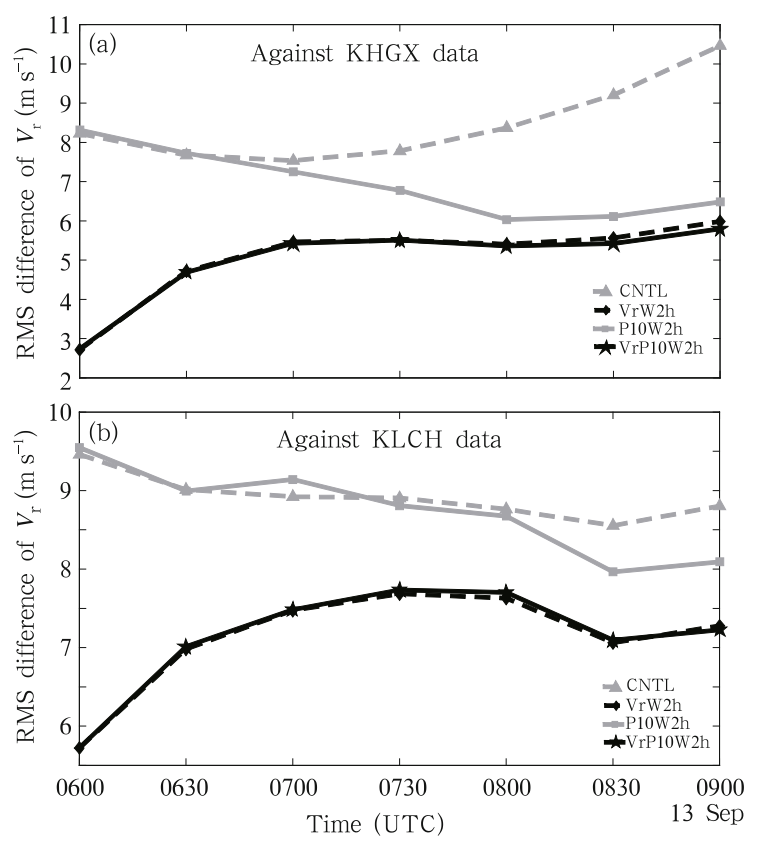

Fig. 9. Root-mean-square differences between observed and forecast $V_{\mathrm{r}}$ for experiments CNTL, VrW2h, P10W2h, and $\mathrm{VrP10W} 2 \mathrm{~h}$, calculated against radar (a) KHGX and (b) $\mathrm{KLCH}$. 
results.

\subsection{Track forecast}

Figure 10 shows the predicted tracks, along with the best track and average track errors, of 18-h forecasts for selected experiments. The forecast vortex center positions are determined by the MSLP and are plotted every $3 \mathrm{~h}$. Despite having a small initial position error of just $7 \mathrm{~km}$, CNTL follows the westernmost track during the 18-h forecast, diverging from all of the DA experiments and the best track (Fig. 10a). The final analyzed vortex centers in the experiments assimilating MSLP are generally closer to the best track than those of CNTL and the experiments assimilating only radar observations. The errors in $\mathrm{P} 10 \mathrm{~W} 2 \mathrm{~h}$, VrP10W2h, and ZP10W2h are 4, 4, and $2.6 \mathrm{~km}$, respectively, comparable to (or even smaller than) the horizontal grid spacing of the model. During the 18$\mathrm{h}$ forecast, the tracks of the experiments assimilating MSLP are similar to each other, except for ZP10W2h, which follows a more westward path at 1800 UTC.

Averaged over the 18-h forecast period, the track errors of P60W2h and P10W2h are generally comparable to those of $\mathrm{VrW} 2 \mathrm{~h}$ (Fig. 10b); the mean error of $\mathrm{P} 60 \mathrm{~W} 2 \mathrm{~h}$ is $2 \mathrm{~km}$ larger than that of $\mathrm{P} 10 \mathrm{~W} 2 \mathrm{~h}$. The track errors of all these three experiments are significantly smaller than the 41-km track error of CNTL (not shown in Fig. 10b). The track errors of VrP60W2h and VrP10W2h are both less than $10 \mathrm{~km}$, smaller than the 12-km error of VrW2h. The track errors of ZP60W2h and ZP10W2h are only $60 \%$ of the error of ZW2h. When MSLP is assimilated with radar observations, the interval of MSLP assimilation (10 vs. $60 \mathrm{~min}$ ) does not have a strong impact on the track; the difference is always smaller than $1 \mathrm{~km}$. In general, most of the experiments assimilating MSLP observations have average track errors of less than $10 \mathrm{~km}$.

Unlike intensity forecast, the track forecast appears to be relatively insensitive to the MSLP assimilation interval. This may be because the initial position errors can be quite effectively corrected in a few MSLP DA cycles, while intensity improvement requires more frequent assimilation cycles to "nudge" and establish a well balanced vortex with sustainable intensity.

\section{Sensitivity of intensity forecast to assimila- tion window length}

In the previous sections, we have shown that during the early analysis and forecast cycles, the MSLP error grows much faster in $\mathrm{P} 10 \mathrm{~W} 2 \mathrm{~h}$ than in $\mathrm{VrW} 2 \mathrm{~h}$ although the error growth rate decreases with time in P10W2h (Fig. 4). Clearly, many MSLP DA cycles help establish a more balanced vortex to slow down the error growth in surface pressure. In this section, we further examine the impact of assimilation window length. Instead of the 2-h assimilation window used in previous experiments, 1 -h or 30-min assimilation window is used, all ending at $0600 \mathrm{UTC}$, and all of them assimilate MSLP and/or $V_{\mathrm{r}}$ data at 10-min intervals.

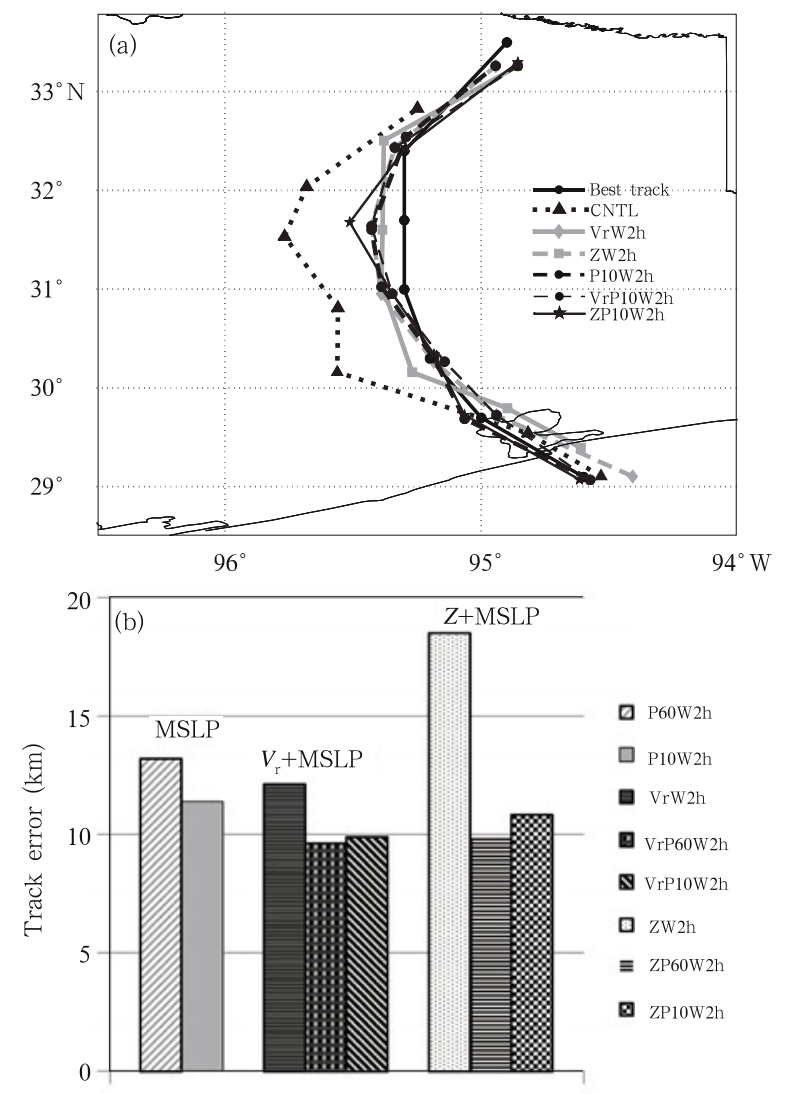

Fig. 10. (a) Forecast TC centers (determined by MSLP position) every $3 \mathrm{~h}$ from 0600 UTC 13 to 0000 UTC 14 September (note that the map is stretched to highlight the difference between the tracks). (b) 18-h average track errors. The average track error of CNTL is $40 \mathrm{~km}$ and not shown here. 
Figure 11 shows predicted MSLPs for the experiments using the three different window lengths, as compared to the CNTL forecast and best track data.

With a $30-\mathrm{min}$ window in $\mathrm{P} 10 \mathrm{~W} 30 \mathrm{~m}$, the analyzed MSLP at $0600 \mathrm{UTC}$ is $953 \mathrm{hPa}$, close to the $950 \mathrm{hPa}$ of best track; it increases by $7 \mathrm{hPa}$ in the first $3 \mathrm{~h}$ of forecast, the largest among all experiments (Fig. 11). In VrW30m, even though the final analyzed MSLP is about $3 \mathrm{hPa}$ higher at 0600 UTC than that in P10W30m, the forecast MSLP at 0900 UTC is actually a couple of hectopascals lower than in P10W30m, and remains lower throughout the $18-\mathrm{h}$ forecast. The assimilation of both MSLP and $V_{\mathrm{r}}$ in VrP10W30m results in a MSLP analysis of about $951 \mathrm{hPa}$, and the forecast values remain a few hectopascals lower than those in P10W30m and VrW30m, closer to the best track values; clearly, assimilating both $V_{\mathrm{r}}$ and MSLP data gives better intensity forecasts than assimilating one of them.

When the assimilation window is extended to 1 $\mathrm{h}$, the general behaviors of the three experiments are similar to the 30-min case, except that the MSLP errors are further reduced by $1-3 \mathrm{hPa}$ in the analyses and forecasts. In comparison, when a 2 -h assimilation win- dow is used, P10W2h actually outperforms VrW2h, analyzing and predicting lower MSLPs that are in better agreement with the best track. VrP10W2h performs slightly better than P10W2h in the MSLP forecast.

Compared to millions of $V_{\mathrm{r}}$ observations available, there is only one MSLP observation at each analysis time. The above results indicate that when using too short assimilation windows, the assimilation of a very limited number of MSLP data is not able to establish a balanced storm as well as the millions of $V_{\mathrm{r}}$ observations can, affecting the surface pressure prediction. The differences in the MSLP forecasts among the three $V_{\mathrm{r}}$ assimilation experiments windows are much smaller, apparently due to the large number of $V_{\mathrm{r}}$ observations, while the forecasts assimilating MSLP data are more sensitive to the window length (Fig. 11). For this particular case, $2 \mathrm{~h}$ appears sufficiently long for the MSLP assimilation performed every $10 \mathrm{~min}$ to produce intensity forecasts comparable to assimilating $V_{\mathrm{r}}$ data.

Assimilating both MSLP and $V_{\mathrm{r}}$ always outperforms the assimilation of one of the observation types, regardless the window length. The improvement is

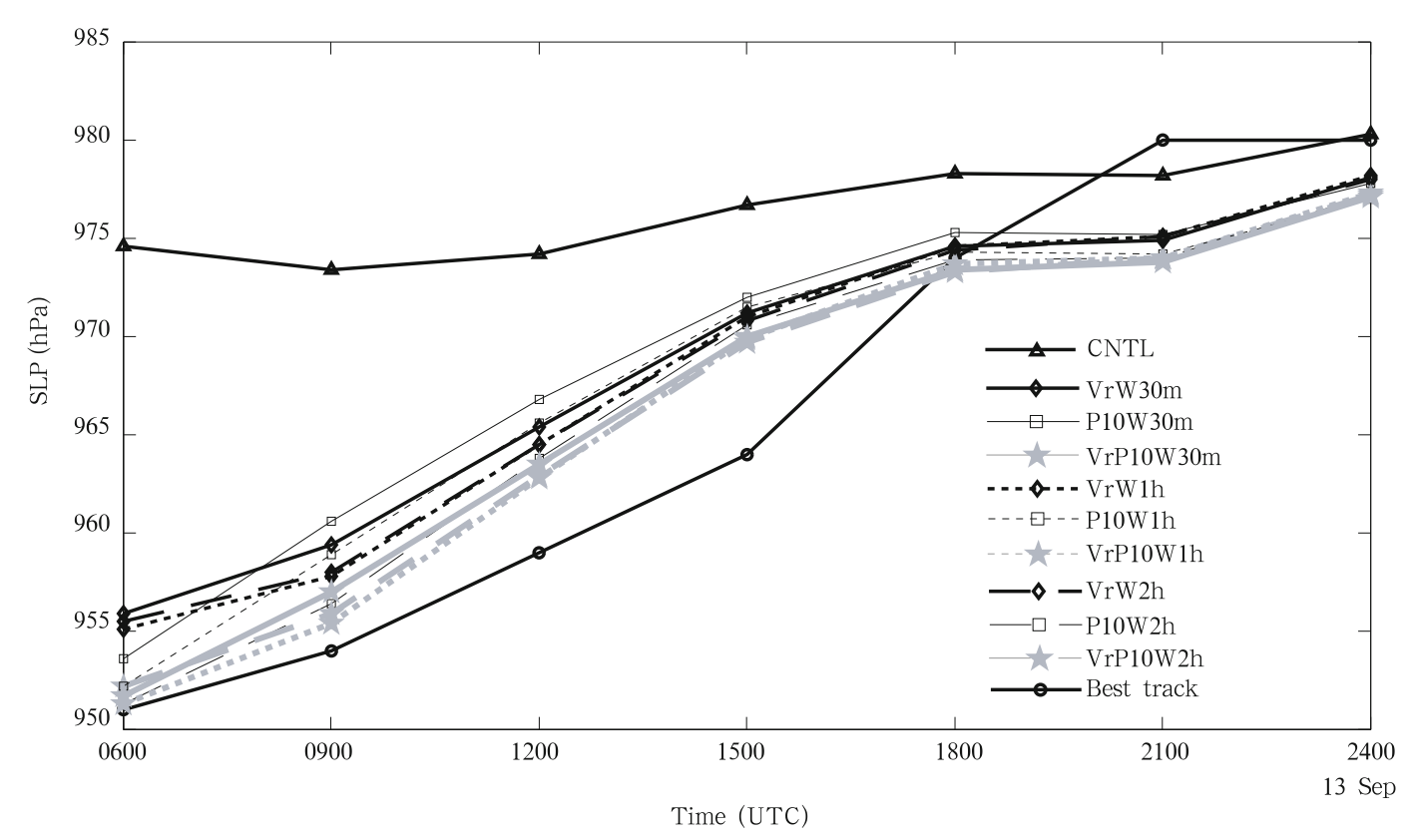

Fig. 11. Forecast minimum sea level pressure from sensitivity experiments varying the assimilation window length, compared to the observed best track and CNTL. 
larger for shorter windows.

\section{Summary and conclusions}

Best track minimum sea level pressure (MSLP) data are treated as surface pressure measurements at TC vortex center and assimilated using an ensemble Kalman filter (EnKF) at a convection-permitting resolution. The study was partly motivated by Hamill et al. (2011) who assimilated TCVital data, including the best track MSLP, into a global forecast model using EnKF; their results showed improved central pressure analyses but the intensity forecast improvement was quickly lost in the subsequent forecasts. The low assimilation frequency and coarse model resolution were believed to be the primary reason. In this study, interpolated MSLP data are assimilated at 60or 10-min intervals for a period of $30 \mathrm{~min}, 1 \mathrm{~h}$, or $2 \mathrm{~h}$ for Hurricane Ike (2008) before it made landfall. The assimilation and forecast experiments used the ARPS model and its EnKF DA system at a 4-km grid spacing. In addition, the relative impacts of MSLP versus those of radar data are examined by assimilating radial velocity $V_{\mathrm{r}}$ and/or reflectivity $Z$ data from two coastal operational radars individually or together with the MSLP data. The radar data are always assimilated at 10-min intervals; the procedure for assimilating radar data using EnKF follows Dong and Xue (2012) exactly.

The first set of experiments examined MSLP DA over a 2 -h window, at 60 - or 10-min intervals. The analysis of MSLP is shown to enhance the hurricane circulation and its warm core structure and it is achieved through cross-variable covariance estimated by the EnKF. Through sensitivity experiments, it is shown that the updating of wind fields when assimilating MSLP data has a more sustainable impact on the intensity forecast than updating temperature, while updating pressure by MSLP has little sustained impact; the model pressure tends to quickly respond to the wind and temperature fields through hydrostatic and gradient wind adjustments, not the other way around. This further highlights the importance of flow-dependent cross-covariance that allows for dy- namically consistent multi-variate analysis of the $\mathrm{TC}$ vortex.

The final analyzed TC vortex is shallower, and its structures are smoother when assimilating MSLP data only while radar data provide more convective-scale structures; this is not surprising because the dense velocity data contain much more convective-scale information while the smaller covariance localization radii used for radar data also help. The analyzed warm core in the former case is also placed too low in the eye region. When MSLP and $V_{\mathrm{r}}$ data are assimilated together, analyses that have better overall vortex intensity and convective-scale structures are obtained.

With 10-min assimilation intervals, the assimilation of MSLP data alone is able to keep the analyzed MSLP lower than that obtained by assimilating $V_{\mathrm{r}}$ data only, but the MSLP forecast error growth is faster than the $V_{\mathrm{r}}$ case, apparently because of the adjustment of the pressure field towards the less wellanalyzed wind and temperature fields. In the case of $V_{\mathrm{r}}$ assimilation, the initially too high MSLP is decreased during the forecast periods, through pressure adjustment towards the better analyzed wind fields. The analyzed MSLP during the later cycles of the 2-h assimilation window is very close to the best track MSLP in the MSLP-assimilation case while that in the $V_{\mathrm{r}}$-only case remains a few hectopascals higher. When both $V_{\mathrm{r}}$ and MSLP are assimilated, errors in both analyzed and forecast MSLPs remain very low in the later cycles.

A 60-min interval when assimilating the MSLP data alone proves insufficient to establish a wellbalanced hurricane vortex in terms of MSLP forecast; the MSLP in the early forecast hours increases much more quickly than the best track (the hurricane was weakening at this stage) while the MSLP error in the experiment assimilating $V_{\mathrm{r}}$ data alone (at 10-min intervals) grows slower (even though the final analyzed MSLP had a slightly larger error). Again, combining MSLP and $V_{\mathrm{r}}$ data has produced the lowest error in the MSLP analysis and forecast. Assimilating reflectivity data alone is able to reduce the MSLP error by only one third in the final analysis relative to the no DA case. 
Using 10-min MSLP assimilation intervals leads to much better MSLP analyses and forecasts than that using 60-min intervals, resulting in lower final MSLP analysis and reduced initial MSLP forecast error growth, and outperforming the forecasts assimilating $V_{\mathrm{r}}$ or $Z$ only. Assimilating MSLP in addition to $Z$ data significantly improves the MSLP analysis and forecast but the improvement in addition to $V_{\mathrm{r}}$ data is much less, because the assimilation of $Z$ data is not very effective at decreasing the MSLP error.

The forecasts are also verified against $V_{\mathrm{r}}$ observations for the first 3 - $\mathrm{h}$ forecast when Hurricane Ike is near the coast. Not surprisingly, the much more voluminous $V_{\mathrm{r}}$ data produce more convective-scale structures than MSLP data and the improved fit of the forecast to $V_{\mathrm{r}}$ observations due to the assimilation of $V_{\mathrm{r}}$ data lasts throughout the 3 -h forecast. The fit to the $V_{\mathrm{r}}$ observations improves over time in the MSLPonly case but the mis-fit remains slightly (about 0.5 $\mathrm{m} \mathrm{s}^{-1}$ ) larger than the $V_{\mathrm{r}}$ case by $3 \mathrm{~h}$.

The assimilation of MSLP also improves the track analysis and forecast. Average 18-h track forecast errors with MSLP assimilation are around 11-13 km, comparable to those obtained using $V_{\mathrm{r}}$ assimilation. Assimilation of MSLP together with $V_{\mathrm{r}}$ or $Z$ improves track forecast more than the assimilation of $V_{\mathrm{r}}$ or $Z$ only.

Sensitivity of the intensity forecast to the length of the MSLP and/or $V_{\mathrm{r}}$ assimilation window is also tested. Using shorter assimilation windows of $30 \mathrm{~min}$ or $1 \mathrm{~h}$ and 10-min assimilation intervals, MSLP forecast with $V_{\mathrm{r}}$ assimilation outperforms the forecast with MSLP assimilation. Using a 2-h window, the opposite is true. For shorter assimilation windows, combining $V_{\mathrm{r}}$ and MSLP gives even more benefits.

In summary, the assimilation of MSLP is able to improve Hurricane Ike analyses and forecasts within a cloud-resolving model, mostly through improvements to the model wind and temperature fields, via crosscovariance of surface pressure with wind and temperature in the EnKF. Because of the very limited pieces of information in the MSLP observations, frequent analyses are necessary to establish a balanced hurricane vortex having slow intensity error growth. Compared to $V_{\mathrm{r}}$ data, MSLP data have less ability in producing convective-scale structures (as verified against $V_{\mathrm{r}}$ observations) and the analyzed warm-core structure is not very realistic. The best results are obtained when $V_{\mathrm{r}}$ and MSLP data are assimilated together.

In our case, the center position of Ike never deviates too far from the best track, and thus the assimilation of MSLP as a regular pressure observation located at the best track position is effective. Such treatment can be problematic if the simulated TC center is far from the best track. The assimilation of TC position and intensity separately (Torn, 2010; Wu et al., 2010) provides a possible solution to this problem.

In order to utilize ground-based radar data, we assimilated MSLP shortly before Ike made landfall. For a rapidly intensifying TC over the ocean, the conclusion about the impact of MSLP assimilation may differ. Further studies with more TCs that are in various stages of development are needed to more completely understand the impacts of MSLP assimilation. Other available observations should also be included in the assimilation to obtain more comprehensive impacts. These can be topics for future studies.

Acknowledgments. Computations were performed in the National Institute of Computational Sciences (NICS) at the University of Tennessee and in the Texas Advanced Computing Center (TACC) at the University of Texas at Austin. Dr. Nathan Snook is thanked for proofreading the manuscript.

\section{REFERENCES}

Benjamin, S. G., and P. A. Miller, 1990: An alternative sea level pressure reduction and a statistical comparison of geostrophic wind estimates with observed surface winds. Mon. Wea. Rev., 118(10), 20992116.

Cangialosi, J. P., and J. L. Franklin, 2011: National Hurricane Center Forecast Verification Report, 77 pp.

Chen, Y. S., and C. Snyder, 2007: Assimilating vortex position with an ensemble Kalman filter. Mon. Wea. Rev., 135(5), 1828-1845.

Dong, J. L., and M. Xue, 2013: Assimilation of radial velocity and reflectivity data from coastal WSR-88D radars using an ensemble Kalman filter for the anal- 
ysis and forecast of landfalling Hurricane Ike (2008). Quart. J. Roy. Meteor. Soc., 139(671), 467-487.

Du, N. Z., M. Xue, K. Zhao, et al., 2012: Impact of assimilating airborne Doppler radar velocity data using the ARPS 3DVAR on the analysis and prediction of Hurricane Ike (2008). J. Geophy. Res., 117, D18113, doi: 10.1029/2012JD017687.

Emanuel, K. A., 2005: Divine Wind: The History and Science of Hurricanes. Oxford University Press, Oxford, 296 pp.

Fovell, R. G., K. L. Corbosiero, and H. C. Kuo, 2009: Cloud microphysics impact on hurricane track as revealed in idealized experiments. J. Atmos. Sci., 66(6), 1764-1778.

,$- \ldots$, A. Seifert, et al., 2010: Impact of cloud-radiative processes on hurricane track. Geoph. Res. Lett., 37, L07808, doi: 10.1029/2010GL042691.

Hamill, T. M., J. S. Whitaker, M. Fiorino, et al., 2011: Global ensemble predictions of 2009's tropical cyclones initialized with an ensemble Kalman filter. Mon. Wea. Rev., 139(2), 668-688.

Houze, R. A., Jr., S. S. Chen, B. F. Smull, et al., 2007: Hurricane intensity and eyewall replacement. Science, 315(5816), 1235-1239.

Huang, X.-Y., 2000: Variational analysis using spatial filters. Mon. Wea. Rev., 128(7), 2588-2600.

Jung, Y. S., G. F. Zhang, and M. Xue, 2008: Assimilation of simulated polarimetric radar data for a convective storm using the ensemble Kalman filter. Part I: Observation operators for reflectivity and polarimetric variables. Mon. Wea. Rev., 136(6), 2228-2245.

—-, M. Xue, and M. J. Tong, 2012: Ensemble Kalman filter analyses of the 29-30 May 2004 Oklahoma tornadic thunderstorm using one- and two-moment bulk microphysics schemes, with verification against polarimetric radar data. Mon. Wea. Rev., 140(5), 1457-1475.

Kurihara, Y., R. E. Tuleya, and M. A. Bender, 1998: The GFDL hurricane prediction system and its performance in the 1995 hurricane season. Mon. Wea. Rev., 126(5), 1306-1322.

Lin, Y.-L., R. D. Farley, and H. D. Orville, 1983: Bulk parameterization of the snow field in a cloud model. J. Climate Appl. Meteor., 22(6), 1065-1092.

Pu, Z. X., and S. A. Braun, 2001: Evaluation of bogus vortex techniques with four-dimensional variational data assimilation. Mon. Wea. Rev., 129(8), 20232039.
—, X. L. Li, and J. Z. Sun, 2009: Impact of airborne Doppler radar data assimilation on the numerical simulation of intensity changes of Hurricane Dennis near a landfall. J. Atmos. Sci., 66(11), 3351-3365.

Purser, R. J., W.-S. Wu, D. F. Parrish, et al., 2003: Numerical aspects of the application of recursive filters to variational statistical analysis. Part I: Spatially homogeneous and isotropic Gaussian covariances. Mon. Wea. Rev., 131(8), 1524-1535.

Rappaport, E. N., J. L. Franklin, L. A. Avila, et al., 2009: Advances and challenges at the National Hurricane Center. Wea. Forecasting, 24(2), 395-419.

Rogers, R., S. Aberson, M. Black, et al., 2006: The intensity forecasting experiment: A NOAA multiyear field program for improving tropical cyclone intensity forecasts. Bull. Amer. Meteor. Soc., 87(11), 1523-1537.

Tong, M. J., and M. Xue, 2008: Simultaneous estimation of microphysical parameters and atmospheric state with simulated radar data and ensemble square root Kalman filter. Part I: Sensitivity analysis and parameter identifiability. Mon. Wea. Rev., 136(5), 1630-1648.

Torn, R. D., 2010: Performance of a mesoscale ensemble Kalman filter (EnKF) during the NOAA High-Resolution Hurricane Test. Mon. Wea. Rev., 138(12), 4375-4392.

—, and G. J. Hakim, 2009: Ensemble data assimilation applied to RAINEX observations of Hurricane Katrina (2005). Mon. Wea. Rev., 137(9), 2817-2829.

Wang, Y. Q., 2002: Vortex rossby waves in a numerically simulated tropical cyclone. Part I: Overall structure, potential vorticity, and kinetic energy budgets. $J$. Atmos. Sci., 59(7), 1213-1238.

—, 2009: How do outer spiral rainbands affect tropical cyclone structure and intensity? J. Atmos. Sci., 66(5), 1250-1273.

Weng, Y. H., and F. Q. Zhang, 2012: Assimilating airborne Doppler radar observations with an ensemble Kalman filter for convection-permitting hurricane initialization and prediction: Katrina (2005). Mon. Wea. Rev., 140(3), 841-859.

Whitaker, J. S., and T. M. Hamill, 2002: Ensemble data assimilation without perturbed observations. Mon. Wea. Rev., 130(7), 1913-1924.

Wu, C.-C., G.-Y. Lien, J.-H. Chen, et al., 2010: Assimilation of tropical cyclone track and structure based on the ensemble Kalman filter (EnKF). J. Atmos. Sci., 67(12), 3806-3822. 
Xue, M., K. K. Droegemeier, V. Wong, et al., 1995: ARPS Version 4.0 User's Guide, 380 pp. Available at http://www.caps.ou.edu/ARPS.

— — - and — , 2000: The Advanced Regional Prediction System (ARPS)-A multi-scale nonhydrostatic atmospheric simulation and prediction model. Part I: Model dynamics and verification. Meteor. Atmos. Physics, 75(3-4), 161-193.

—,- - _ - et al., 2001: The Advanced Regional Prediction System (ARPS)-A multi-scale nonhydrostatic atmospheric simulation and prediction tool. Part II: Model physics and applications. Meteor. Atmos. Phys., 76(3-4), 143-165.

—, D.-H. Wang, J.-D. Gao, et al., 2003: The Advanced Regional Prediction System (ARPS), storm-scale numerical weather prediction and data assimilation. Meteor. Atmos. Phys., 82(1-4), 139-170.

-, M. J. Tong, and K. K. Droegemeier, 2006: An OSSE framework based on the ensemble square root Kalman filter for evaluating the impact of data from radar networks on thunderstorm analysis and forecasting. J. Atmos. Ocean Tech., 23(1), 46-66.
Zhang, F. Q., Y. H. Weng, J. A. Sippel, et al., 2009: Cloud-resolving hurricane initialization and prediction through assimilation of Doppler radar observations with an ensemble Kalman filter. Mon. Wea. Rev., 137(7), 2105-2125.

Zhao, K., and M. Xue, 2009: Assimilation of coastal Doppler radar data with the ARPS 3DVAR and cloud analysis for the prediction of Hurricane Ike (2008). Geophys. Res. Lett., 36(12), L12803.

— X. X. F. Li, M. Xue, et al., 2012: Short-term forecasting through intermittent assimilation of data from Taiwan Region and Mainland China coastal radars for Typhoon Meranti (2010) at landfall. J. Geophy. Res., 117, D06108, doi: 10.1029/2011JD017109.

Zhao, Q. Y., and Y. Jin, 2008: High-resolution radar data assimilation for Hurricane Isabel (2003) at landfall. Bull. Amer. Meteor. Soc., 89(9), 1355-1372.

Zhu, T., and D.-L. Zhang, 2006: Numerical simulation of Hurricane Bonnie (1998). Part II: Sensitivity to varying cloud microphysical processes. J. Atmos. Sci., 63(1), 109-126. 